\title{
Feasibility Study on the New Structure of a Spindle Motor for Hard Disk Drive
}

\author{
Tae-Woo Kim *, and Jung-Hwan Chang *
}

\begin{abstract}
This paper presents the new structure of a spindle motor for hard disk drive (HDD). It can produce axial force as well as torque without a pulling plate or a pulling magnet required for the normal operation of a hydrodynamic bearing in rotating-shaft structure. The proposed models have different air gap length along the axial direction by changing the thickness of permanent magnet (PM). One has a single slope and the other has double slopes on the surface of PM. For the design of the proposed models, variables are defined and its effects on the motor performances are investigated by 3-demensional finite element analysis (FEA). The equi-performance curves are investigated for the main characteristics of the spindle motor such as generated torque, axial force and torque ripple ratio. The validity of the proposed models is verified by the feasibility study and performance evaluation.
\end{abstract}

Keywords: Axial force, Hard disk drive, Hydrodynamic bearing, Pulling magnet, Pulling plate, Spindle motor

\section{Introduction}

A spindle motor for hard disk drive (HDD) operates in high speed and especially needs high efficiency and robust dynamics. A hydrodynamic bearing is generally used for this motor due to its excellent dynamic characteristics [1][2]. However, in a rotating-shaft structure, it requires constant axial force for its normal operation unlike a ball bearing and thus it needs additional structure to produce the axial force. In a conventional model, two different methods are generally used. One method is adopting a pulling plate at the stationary part under the rotating permanent magnet (PM) and the other method is applying a pulling magnet at the inner part of stator core beneath the rotating hub [3]-[4]. Both of them generates the axial force for pulling down the rotating bodies and improves the dynamic characteristics of the spindle motor. However, they also make leakage flux, considerable iron losses and increase manufacturing cost as well.

This paper presents two models producing required torque and axial force without additional magnetic structure such as the pulling plate or the pulling magnet. They have different air gap length along the axial direction by

\footnotetext{
Dept. of Electrical Engineering, Dong-A University, Korea. (cjhwan@dau.ac.kr)

Received 10 July 2013; Accepted 20 August 2013
}

changing the thickness of PM. The first model has a single slope and the second model has double slopes on the surface of PM. The design variables for the proposed models are defined and its effects are analyzed by 3demensional finite element analysis (FEA). The equiperformance curves are investigated for the main characteristics of the spindle motor such as generated torque, axial force and torque ripple ratio. The validity of the proposed model is verified by the feasibility study and performance evaluation.

\section{New Structure of a Spindle Motor}

\subsection{Conventional Model}

Fig. 1 shows the conventional model for a $2.5 \mathrm{in}$. HDD spindle motor. The rotating part is consist of PM and hub and the stationary part is consist of stator core, coil, base plate and pulling plate to produce axial force by the interaction with the rotating PM. The spindle motor has 12 poles 9 slots and runs 7,200 rpm with the specifications as shown in Table 1. For studying the effects of the pulling plate on the motor performances, the magnetic field is analyzed by 3 -dimensional FEA using the commercial software, FLUX 3D. 


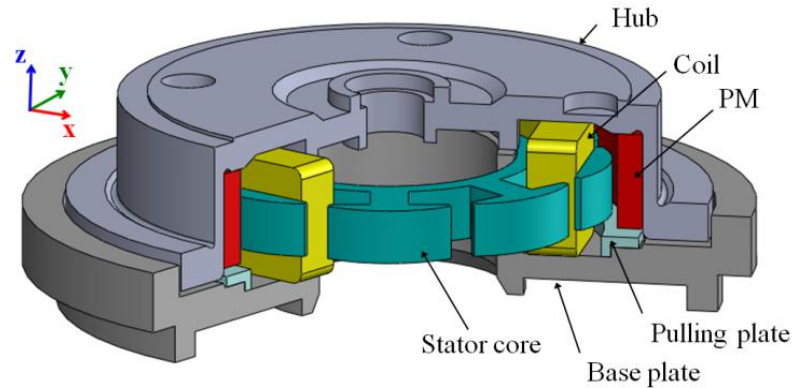

Fig. 1. Structure of a spindle motor for HDD

Table 1. Specifications of the conventional spindle motor

\begin{tabular}{|c|c|c|}
\hline \multicolumn{2}{|c|}{ Quantity } & Value \\
\hline \multirow{3}{*}{$\begin{array}{c}\text { General } \\
\text { Data }\end{array}$} & Rated speed [rpm] & 7,200 \\
\cline { 2 - 3 } & Rated torque $[\mu \mathrm{Nm}]$ & 674 \\
\cline { 2 - 3 } & Air gap length [mm] & 0.25 \\
\hline \multirow{3}{*}{ Stator } & Number of slots & 9 \\
\cline { 2 - 3 } & Number of turns & 57 \\
\cline { 2 - 3 } & Outer diameter [mm] & 16.5 \\
\hline \multirow{4}{*}{ Rotor } & Number of pole & 12 \\
\cline { 2 - 3 } & Remanence of PM $[\mathrm{T}]$ & 0.7 \\
\cline { 2 - 3 } & Thickness of PM [mm] & 0.95 \\
\cline { 2 - 3 } & Outer diameter [mm] & 20.0 \\
\hline
\end{tabular}

Fig. 2 compares the axial force and generated torque with and without the pulling plate. The model without the pulling plate generates axial force of $0.2 \mathrm{~N}$ acting downward on the rotating parts due to the asymmetrical PM overhang length in the axial direction. However, this is not enough force for the stable operation of hydrodynamic bearing and causes to installation of another magnetic structure to satisfy the required axial force, which is in the range between $0.8 \mathrm{~N}$ and $1.2 \mathrm{~N}$. In case of having the pulling plate, the spindle motor produces $0.9 \mathrm{~N}$ axial force by additional magnetic pull between PM and the pulling plate.
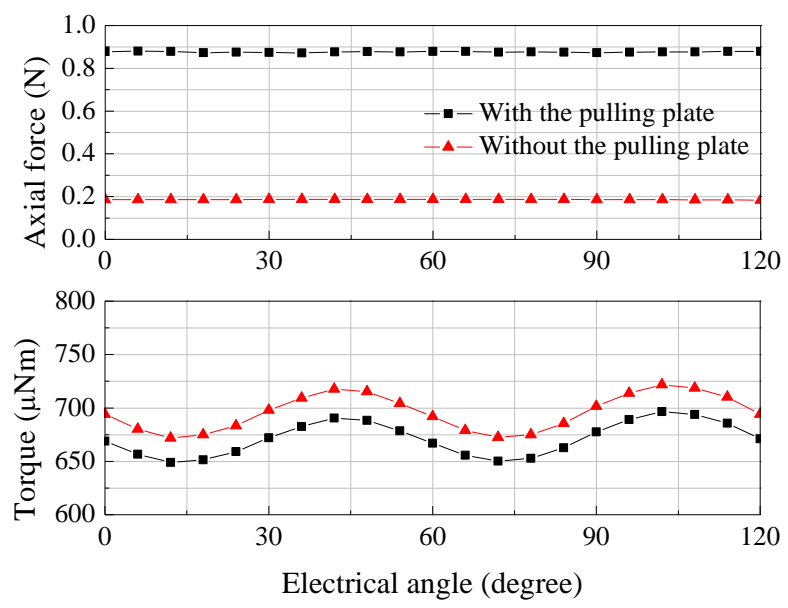

Fig. 2. Comparison of axial force and generated torque with and without the pulling plate
Contrary to this positive role of the pulling plate, there are other things to be considered in adopting it. The first one is the leakage flux. It reduces the mutually coupled magnetic flux between PM and stator core and decreases the generated torque by $4 \%$ as shown in Fig. 2.

The other important thing is the time-varying magnetic flux on the stationary pulling plate, which has nonlaminated structure. It produces additional iron losses. In the frequency domain, the iron loss model developed by Berotti is widely used [5]. According to the model, the specific iron loss can be separated into hysteresis loss $P_{h}$, classical eddy current loss $P_{c}$ and excess loss $P_{e}$ as follows;

$$
P_{\text {loss }}=P_{h}+P_{c}+P_{e}=k_{h} f B_{m}^{\alpha}+k_{c} f^{2} B_{m}^{2}+k_{e} f^{1.5} B_{m}^{1.5}
$$

where $f$ and $B_{m}$ are the frequency and peak magnetic flux density respectively. The coefficients $k_{h}, k_{c}, k_{e}$ and $\alpha$ are determined from the measured data for a certain frequency. Given the coefficients, the total iron loss is calculated in terms of the frequency and peak magnetic flux density. Based on the iron loss model of Berotti, the model having pulling plate has $48 \%$ increased iron losses at the stationary parts as shown in Table 2.

Table 2. Comparison of iron loss based on Berotti model

\begin{tabular}{|c|c|c|}
\hline Iron loss & $\begin{array}{c}\text { With the Pulling } \\
\text { plate }\end{array}$ & $\begin{array}{c}\text { Without the } \\
\text { pulling plate }\end{array}$ \\
\hline Stator $[\mathrm{mW}]$ & 61.17 & 66.71 \\
\hline $\begin{array}{c}\text { Pulling plate } \\
{[\mathrm{mW}]}\end{array}$ & 37.63 & - \\
\hline Total $[\mathrm{mW}]$ & 98.80 & 66.71 \\
\hline
\end{tabular}

\subsection{Proposed Model}

Fig. 3 shows the cross-sectional views of the conventional and proposed models. The one of the main differences between them is the uniformity of the air gap length. Unlike the conventional model, the proposed models have different air gap length in the axial direction. The model I has a single slope on the surface of the PM as shown in Fig. 3 (b). This kind of non-uniform air gap length contributes to generate axial force as well as torque. The main design variables are the slope angle, $\theta$ and overhang length of PM, $h_{1}$. In order to apply the slope angle as a variable, the air gap length, $g$ on the upper part of stator is fixed as $0.2 \mathrm{~mm}$. Although the increased slope angle and the reduced overhang length have positive influence on the axial force, this decrease the generated torque. Thus, in model I, the remanence of PM also should be included in 
the design variables to generate torque in the required range.

The other proposed model, model II, has double slopes on the surface of PM as shown in Fig. 3 (c). It has a tilted surface ending at the one point on the PM region facing stator teeth. Compared to the model I, it has somewhat increased average air gap length and can reduce the leakage flux. With this benefit, model II can meet the required performances without considering higher grade of PM. In model II, the slope angle, $\theta$ and the PM length from the upper end face of the stator to the starting point of straight part in the PM surface are selected as the main design variables.

The other important difference between models is the direction of magnetization of PM. As shown in Fig. 4, it is perpendicular to the tilted inner surface of PM. This also contributes to generate the axial force.
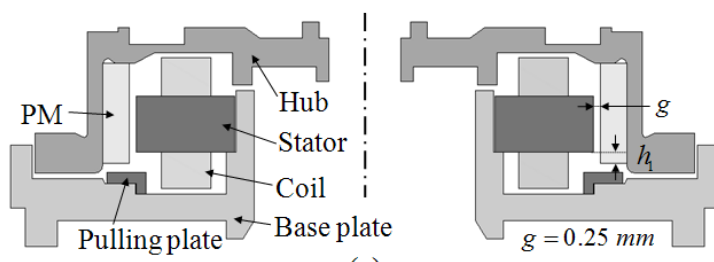

(a)

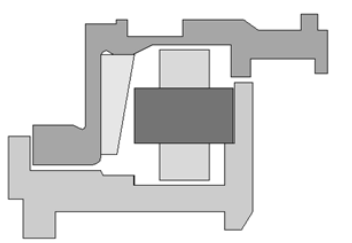

(b)
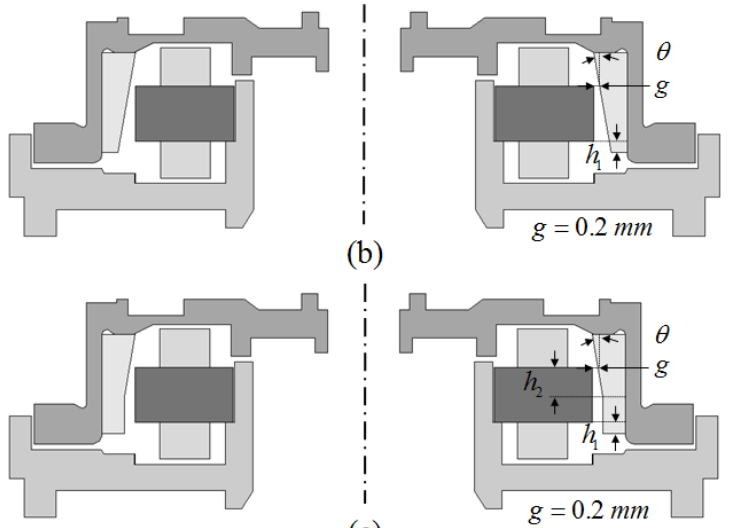

)

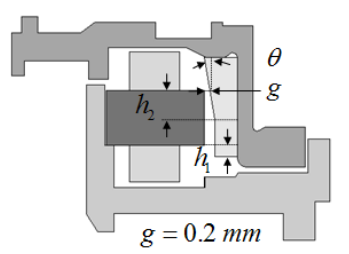

(c)

Fig. 3. Structures of the spindle motor for HDD (a) conventional model (b) proposed model I (c) proposed model II

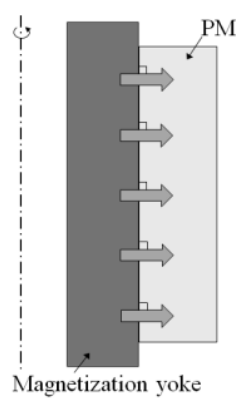

(a)

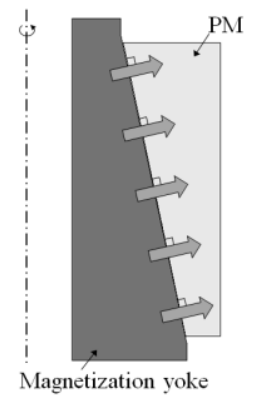

(b)

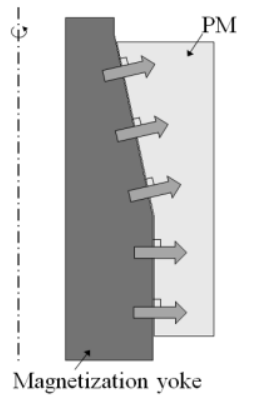

(c)
Fig. 4. Direction of the magnetization (a) conventional model (b) proposed model I (c) proposed model II

\section{Feasibility Study}

With the variation of the design variables of the proposed models, the equi-performance curves are investigated for the main characteristics of the spindle motor such as generated torque, axial force and torque ripple ratio. In the conventional model, the value of the axial force, generated torque and torque ripple ratio are $0.9 \mathrm{~N}, 674 \mu \mathrm{Nm}$ and $5.9 \%$ respectively. By the reference with these values, the feasible ranges of the axial force, generated torque and torque ripple ratio are determined as shown in Table 3.

Table 3. Feasible range of the axial force, generated torque and torque ripple ratio

\begin{tabular}{|c|c|}
\hline Variables & Feasible range \\
\hline Axial force $[\mathrm{N}]$ & $0.8 \sim 1.0$ \\
\hline Generated torque $[\mu \mathrm{Nm}]$ & $670 \sim 690$ \\
\hline Torque ripple ratio $[\%]$ & $5 \sim 7$ \\
\hline
\end{tabular}

\subsection{Proposed Model I}

Fig. 5 shows the equi-performance curves of the model I for the axial force, generated torque and torque ripple ratio with the variation of the $h_{1}$ and $\theta$. In case of model I, it is not possible to satisfy all of the reference performance listed in Table III by using the same grade of PM with the conventional model. The remanence of PM is increased to $0.78 \mathrm{~T}$, which is the least value to have a feasible region on the design of the spindle motor. As expected, with the increase of the lower overhang length of PM, $h_{1}$, the axial force is decreased and the generated torque is increased for the same slop angle. On the other hand, for the fixed $h_{1}$, the axial force is increased and the generated torque is decreased as the slope angle is getting bigger. For the two design variables, the axial force is inversely proportional to the generated torque. In case of torque ripple ratio, it has generally smaller value with increased overhang length and reduced slop angle. By considering both of characteristics, the feasible region satisfying reference values can be obtained as shown in Fig. 5 (d).

\subsection{Proposed Model II}

Fig. 6 shows the equi-performance curves of the model II for the axial force, generated torque and torque ripple ratio with the variation of the $h_{2}$ and $\theta$. The value of $h_{1}$ is fixed to $0.13 \mathrm{~mm}$ by referring the results of the proposed model I. Unlike the model I, it can satisfy the reference performances without increase of the remanence of the PM by adopting double slopes on the surface of PM. 


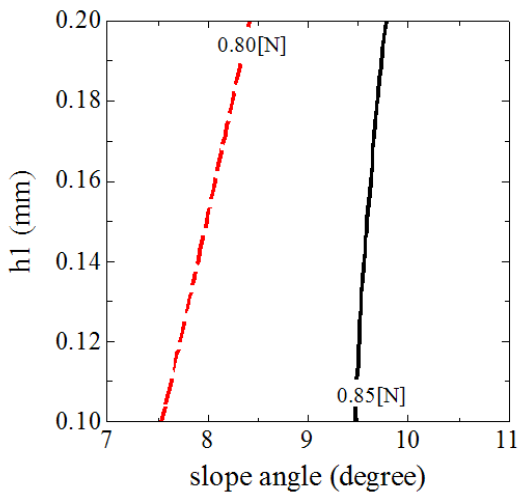

(a)

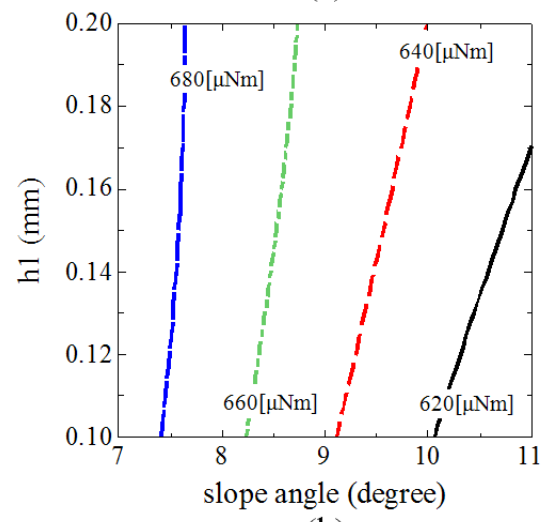

(b)

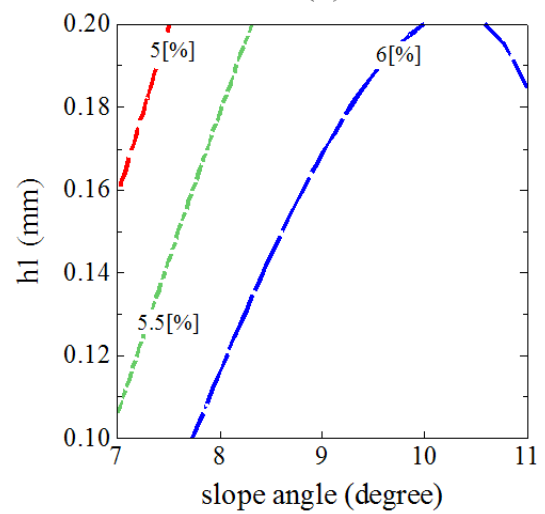

(c)

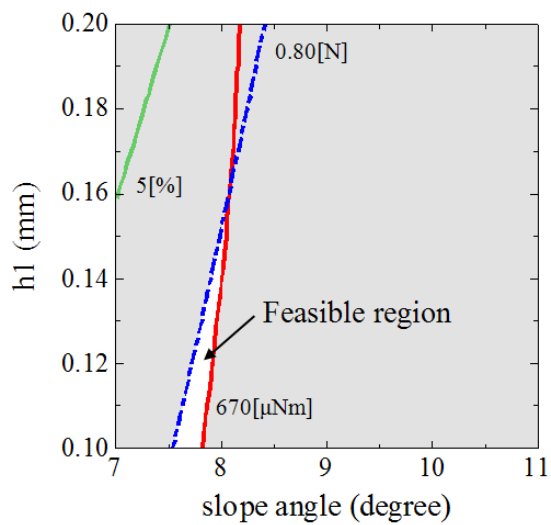

(d)

Fig. 5. Equi-performance curves of the proposed model I (a) axial force (b) generated torque (c) torque ripple ratio (d) feasible region

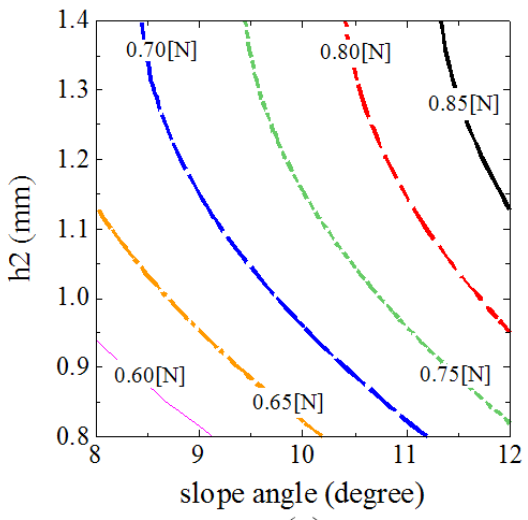

(a)

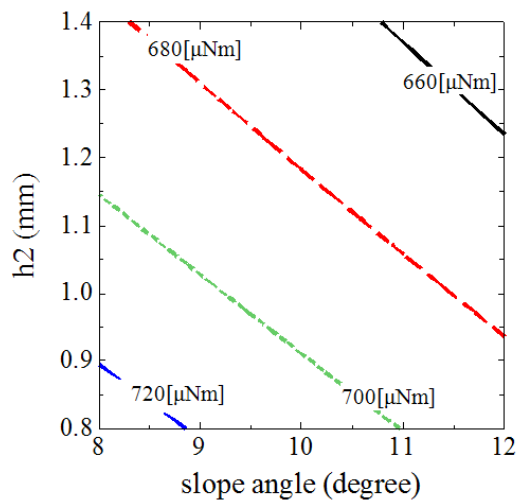

(b)

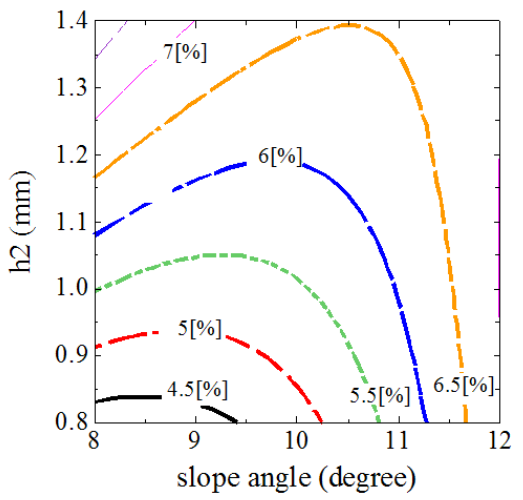

(c)

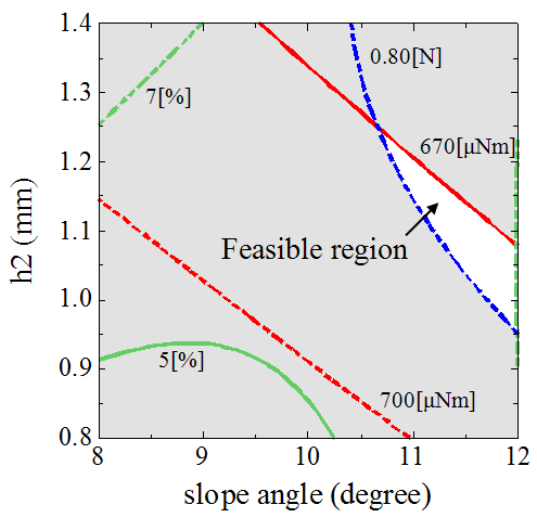

(d)

Fig. 6. Equi-performance curves of the proposed model II (a) axial force (b) generated torque (c) torque ripple ratio (d) feasible region 
With the increase of the $h_{2}$ meaning the decrease of the straight part of PM, the axial force is increased and the generated torque is decreased for the same slope angle. . In case of torque ripple ratio for the fixed $h_{2}$, it has a concave shape with the increase of the slope angle. By combining the relationship as done in model $\mathrm{I}$, the feasible region can be also obtained as shown in Fig. 6 (d). In both cases, the feasible solutions are available for the design of the spindle motor for HDD without additional structure to produce the axial force.

\section{Conclusion}

This paper proposes the new structure of a spindle motor for HDD using hydrodynamic bearing. The proposed models have different air gap length by adopting single and double slopes on the surface of PM. By changing the uniform air gap length, the proposed models generate the required torque and axial force without the additional structure such as a pulling plate or a pulling magnet. With these new structures, the efficiency of the spindle motor can be improved by eliminating iron losses due to the pulling plate or the pulling magnet. The further study shall be done by the prototyping of the proposed model with optimization and experiment.

\section{References}

[1] G. H. Jang and J. S. Park, "Development of a highly efficient hard disk drive spindle motor with a passive magnetic thrust bearing and a hydrodynamic journal bearing," Journal of Applied Physics, vol. 97, pp. 10Q507 - 10Q507 - 3, May. 2005.

[2] T. Asada, H. Saitou, Y. Asaida and K. Itoh, "Characteristic analysis of hydrodynamic bearings for HDDs," IEEE Trans. Magn, vol. 37 pp. 810-814, Mar. 2001.

[3] Vladimir V. Popov, "Permanent magnet motor having an axially magnetized pull magnet," U.S. Patent 03091 84, Dec 18,2008

[4] Samsung Electro-Mechanics Co, "Spindle motor with hydrodynamic pressure bearing," K.R. Patent 00329 55, Mar 30, 2011

[5] Bertotti G. "General properties of power losses in soft ferromagnetic materials," IEEE Trans. Magnetics, vol. 24, no. 1, pp. 621-630, Jan. 1988.

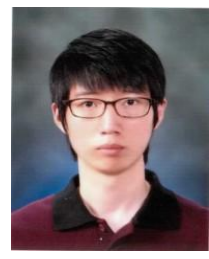

Tae-Woo Kim received B.S. and M.S. degrees in electrical engineering from Dong-A University, Busan, Rep. of Korea in 2011 and 2013, respectively. $\mathrm{He}$ is currently working toward the Ph.D. degree at the same University. His research interests are the analysis and design of permanent magnet synchronous motor.

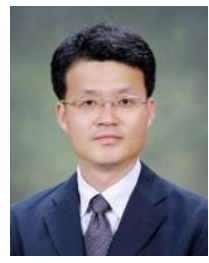

Jung-hwan Chang received B. S. and M.S. degrees in electrical engineering and Ph.D. degree in precision mechanical engineering from Hanyang University, Seoul, Rep. of Korea in 1994, 1997 and 2001, respectively. From 2001 to 2002, he worked at Institute of Brain Korea 21 at Hanyang University, where he developed micro drive and high-speed spindle motor. From 2002 to 2003, he worked as research fellow at University of California at Berkeley with the support of Korea Science and Engineering Foundation, and analyzed and developed electrically controlled engine valve system. From 2003 to 2009, he worked in Korea Electrotechnology Research Institute (KERI) as a senior researcher, and engaged in the developments of special purpose machines. Since 2009, he has been with the department of electrical engineering, Dong-A University, Busan Rep. of Korea, as an associate professor. His interests are the design and analysis of electro-mechanical systems including driving circuits. 